\title{
Understanding the balance of entropy and enthalpy in hydrogen-halide noncovalent bonding
}

\author{
David Bodesheim, Gregor Kieslich, Mike Johnson, \\ and Keith T. Butler
}

\section{Published version information}

Citation: D Bodesheim et al. "Understanding the balance of entropy and enthalpy in hydrogen-halide noncovalent bonding." Journal of Physical Chemistry Letters, vol. 11, no. 9 (2020): 3495-3500.

DOI: $10.1021 /$ acs.jpclett.0c00817

This document is the unedited author's version of a Submitted Work that was subsequently accepted for publication in Journal of Physical Chemistry Letters copyright (C2020 American Chemical Society, after peer review. To access the final edited and published work see DOI above.

Please cite only the published version using the reference above. This is the citation assigned by the publisher at the time of issuing the AAM. Please check the publisher's website for any updates. 


\title{
Understanding the Balance of Entropy and Enthalpy in Hydrogen-Halide Non-Covalent Bonding
}

\author{
David Bodesheim \\ Department of Chemistry, Technical University of Munich, \\ Lichtenbergstrasse 4, D-85748 Garching, Germany. \\ Gregor Kieslich \\ Department of Chemistry, Technical University of Munich, \\ Lichtenbergstrasse 4, D-85748 Garching, Germany \\ Mike Johnson \\ ISIS Facility, Rutherford Appleton Laboratory, Harwell Oxford, Didcot, Oxfordshire OX11 OQX, UK. \\ Keith T. Butler* \\ Scientific Computing Department, Rutherford Appleton Laboratory, \\ Harwell Oxford, Didcot, Oxfordshire OX11 OQX, UK.
}

(Dated: April 10, 2020)

\begin{abstract}
Hydrogen bonds are of great scientific interest, determining the free energy landscape and hence chemical and physical properties of many materials systems, for example the hybrid organic inorganic perovskites. Although these interactions are critical, understanding them is difficult in complex, multi-component systems; hydrogen halides are ideal as simple binary model compounds to understand the role of hydrogen bonding in physical properties like phase transitions. Here we investigate the orthorhombic low-temperature phase and the cubic high-temperature phase in $\mathrm{HX}(\mathrm{X}=\mathrm{F}, \mathrm{Cl}, \mathrm{Br}, \mathrm{I})$ systems understanding how different hydrogen-halide bonds influence free energy profiles. We show that hydrogen fluoride has a qualitatively different behaviour due to strong hydrogen bonding and hence very different vibrational entropy. Heavier halides are in contrast rather similar in their physical properties, however, dispersion interactions play a more crucial role in these. These results have implications for rational design of materials with hydrogen-halide bonds and tuning material properties in systems like mixed anion $\mathrm{CH}_{3} \mathrm{NH}_{3} \mathrm{PbX}_{3}$ perovskites.
\end{abstract}

\section{INTRODUCTION}

The growing scientific interest in dense and porous coordination polymers with tailored degrees of structural flexibility has reminded us about the importance of supposedly weak interactions such as hydrogen bonds and dispersion forces. [1] Understanding their impact on the free energy landscape of stimuli responsive materials is as challenging as it is important, providing experimentalists

\footnotetext{
*keith.butler@stfc.ac.uk
}

with an entry door for tuning the responsive behaviour of materials to external stimuli such as temperature, pressure or guest molecules. [2] Examples in which the balance between these interactions accounts for the temperature and pressure responsive properties span from ferroelectrics and barocalorics to optoelectronics. For instance, the ferroelectric phase transition in $\mathrm{KH}_{2} \mathrm{PO}_{4}$ is related to the formation and loss of hydrogen bonds [3] and the rich phase evolution in the photovoltaic absorber material $\left[\mathrm{CH}_{3} \mathrm{NH}_{3}\right] \mathrm{PbBr}_{3}$ originates from an interplay between hydrogen bonds, dispersion interactions and entropic effects[4]. Likewise, the large network flexibility of some MOFs as a response to an external stimulus involves a complex free energy landscape where dispersion interactions have found to play an important role.[5, 6]

Recent advances in terahertz spectroscopy and computational materials science have combined to being to highlight the critical role played by vibrations in the structure and properties of all solids, but in particular those with intermolecular interactions. [7] For example, the evolution of mechanical properties of MOF-5 under pressure can be tracked by combined theory and experiment [8]. Critical insights into the balance between entropy and enthalphy have also recently been provided by the application of state-of-the-art desnity functional theory (DFT) calculations on size dependent phase stability is soft porous nanocrystals. And the study of dynamical properties of MOFs using lattice[9] and molecular[10] dynamics has provided insights into the structure and chemistry of MOFs. Nonetheless, the complexity of parameters which includes periodic and dynamic aspects such as hydrogen bonding interactions and contributions from vibrational and configurational entropy challenges experimentalists and computational scientists.[11-13]

Given this complexity, we decided to go one step back and revisit the parameters that determine the thermodynamics of one of the arguably simpler but yet technologically relevant systems: the binary acids HX 
with $\mathrm{X}=\mathrm{F}, \mathrm{Cl}, \mathrm{Br}$ and I. In their solid forms these compounds fulfill the definition of a hydrogen bonded system with $\mathrm{X}-\mathrm{H} \cdots \mathrm{A}$ moieties with $\mathrm{A}=\mathrm{X}^{\prime}$.[14] These systems also provide an unambiguous "toy model" in which to understand how free energy in non-covalent hydrogen halide bonds evolves with temperature; an important factor in understanding composition-structure-property relationships in hybrid organic inorganic perovskites (HOIPs).

The binary hydrogen halides have been in the focus of various crystallographic and spectroscopic studies since the 1930s. HF crystallises in an orthorhombic Bmb2 ${ }_{1} \mathrm{~b}$ structure (space group 36, standard notation: $\mathrm{Cmc} 2_{1}$ ) and shows no evidence of a phase transitions until melting at $\mathrm{T}=189.75 \mathrm{~K}$. $[15,16]$ In solid HF, the dominating structural feature are parallel zig-zag chains of hydrogen halide units, see Figure 1a. When comparing $\mathrm{HCl}$ and $\mathrm{HBr}$ to $\mathrm{HF}$, it can be observed that both have the same low-temperature groundstate structure as HF. [17, 18] Interestingly, $\mathrm{HBr}$ and $\mathrm{HCl}$ both have a cubic high-temperature phase with space group Fm3m (225), which can be considered to be an average structure with 12-fold disorder of the $\mathrm{H}$ sites due to studies on both $\mathrm{DCl}$ and $\mathrm{DBr}$ see Figure $1 \mathrm{~b}$. In $\mathrm{HCl}$, the orthorhombic to $\mathrm{cu}-$ bic phase transitions occurs at $98.4 \mathrm{~K}$. [17, 19-23] Moreover, $\mathrm{HBr}$ exhibits two additional phase transitions to an orthorhombic Bbcm (64) and cubic phase Pa $\overline{3}$ (205), with transition temperatures of $116.9 \mathrm{~K}$ (225 to 205$), 113.6 \mathrm{~K}$ (205 to 64$)$ and $89.7 \mathrm{~K}$ (64 to 36$).[23,24] \mathrm{In} \mathrm{HI}$, three crystallographically distinct phases have been observed in the past. Neutron-experiments on DI and spectroscopic investigations of HI suggest a triclinic structure ground low temperature structure $(\mathrm{P} \overline{1}, 2)$ with stacked layers of hydrogen-bonded HI squares.[25, 26] In turn, HI exhibits two phase transitions at $125.7 \mathrm{~K}$ (225 to 64$)$ and at $70.1 \mathrm{~K}$ (64 to 2).[23, 24] Figure 1c) summarizes the different phases in hydrogen halides that have been reported until today.

In this paper we explore and rationalise the thermodynamic factors and microscopic origin of the observed trends in phase transition temperatures of hydrogen halides using density functional theory (DFT) calculations. We begin by considering the bonding enthalpy and understanding how the role of dispersion differs among the halides. By applying lattice dynamic calculations, we provide an in-depth picture of the underlying free energy landscape and the chemistry of the different $\mathrm{HX}$ compounds with $\mathrm{X}=\mathrm{F}, \mathrm{Cl}, \mathrm{Br}$ and I. For the sake of clarity of comparison, we only consider the orthorhombic low-temperature phase and the cubic hightemperature phase as model system. We then discuss the general trends which emerge from our analysis and briefly discuss these findings in the context of HOIP design. a)

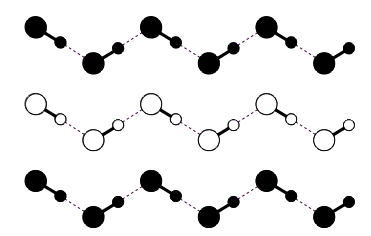

b)

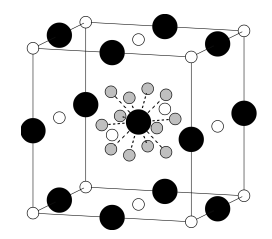

c)

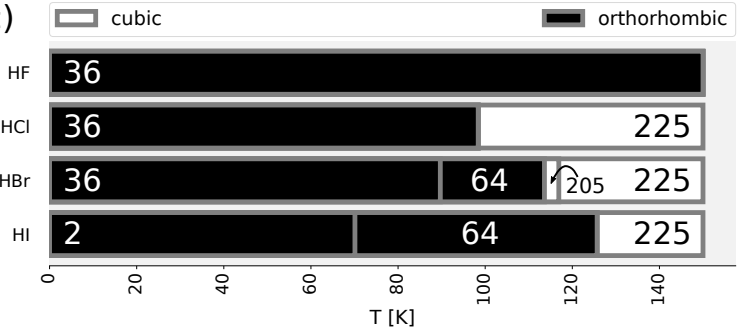

FIG. 1. a) Chain structure of the 36 phase. Black depicts the atoms in the front-plane and white the atoms in the backplane. Big atoms are halides, small are hydrogen. The dashed line indicates hydrogen bonds. b) Crystal structure of the 225 phase. Black depicts halide and white hydrogen atoms. The hydrogen in this system is seen as 12-fold disordered which is demonstrated as an example for the central chlorine atoms by the grey hydrogen atoms situated along the lines to the 12 nearest neighbours. c) Summary of the different phases in solid hydrogen halides. Numbers indicate space groups.

\section{RESULTS AND DISCUSSION}

In a first step, we calculated the hydrogen bonding energy $E_{\text {Bonding }}$ for the orthorhombic low-temperature structure (36) and the cubic high-temperature structure (225) for all compounds. For obtaining $E_{\text {Bonding }}$, an HX unit was removed from the relaxed unit cell of the orthorhombic and cubic structures which were taken from the literature, see Table S2. In a next step, single-point calculations for the unit-cell with one removed HX unit and the unit-cell with one single HX unit were performed. Subsequently, $E_{\text {Bonding }}$ was calculated by:

$$
E_{\text {Bonding }}=E_{N}-\left(E_{H X-\text { removed }}+E_{H X-\text { single }}\right)
$$

where $E_{N}$ is the calculated energy of the pristine system, $E_{H X \text {-removed }}$ is the energy of that system with one HX unit removed and $E_{H X-\text { single }}$ is the energy of the lone HX unit. This methodology was recently developed by a few of us to calculate the hydrogen bonding energy for hybrid inorganic-organic perovskites.[27] To quantify the influence of dispersion interactions, the same single-point calculations were performed with the previously used optimised structure, but without using a van-der-Waals (vdW) correction and without re-optimising the structure. The resulting hydrogen bonding energies are shown in Figure 2, revealing an increasing importance of dispersive interactions when going along the series $\mathrm{HF}, \mathrm{HCl}, \mathrm{HBr}$ to $\mathrm{HI}$. Interestingly, $\mathrm{HF}$ seems to behave significantly different when compared to the heavier homologues. In HF, the contribution from dispersion 

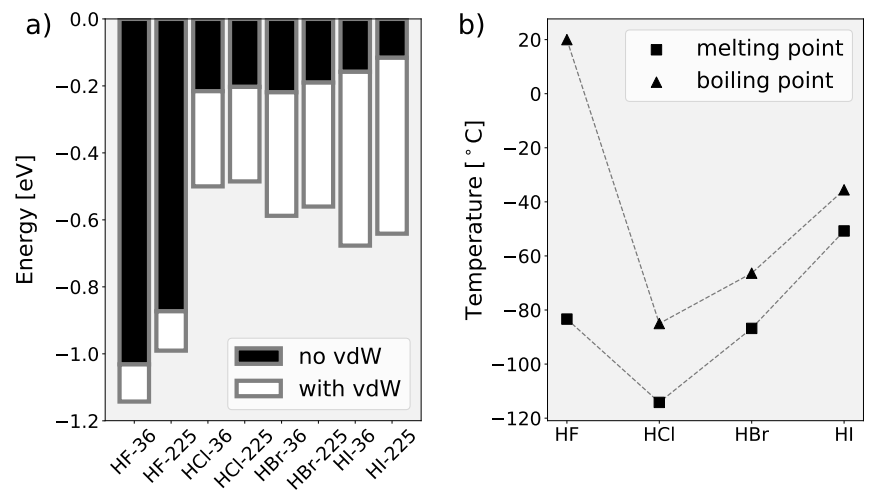

FIG. 2. a) Hydrogen-bonding energies in the different HXsystems and their phases with and without vdW-correction. Black is the energy without vdW-correction, black+white is the energy with vdW-correction. b) Melting and boiling points of the hydrogen halide systems (data from [28]).

interactions is only a small component to the total energy, whilst in all other HX system it constitutes at least $50 \%$. The contribution from dispersion increases as the halide becomes more polarisable, reflecting the chemists intuition in estimating the character of a chemical bond. Additionally, the general trend in hydrogen bonding energies is correlated with the trends of melting and boiling points of the HX systems, see Figure 2b, nicely highlighting the importance of hydrogen bonding interactions.

In the bigger picture, a different behaviour of HF compared to the other homologues can be observed. The different nature of dispersion interactions in $\mathrm{HF}$ is a first clue to the origins of this, but as we will see, a dynamic will reveal additional insight. Therefore, we now move beyond a static lattice picture and study the vibrational properties of the HX systems to rationalise the thermodynamic factors that determine the free energy landscape of these systems and in turn the phase transitions energetics. We will begin by calculating the phonon density of states (pDoS) and the resulting quantities such as the vibrational entropy $\left(S_{v i b}\right)$ and the Gibbs free energy $(G)$. The calculated $G$ follows the same trend as observed experimentally, i.e. HF has no phase transition, and the transition temperature from orthorhombic to cubic increases in the order $\mathrm{Cl}<\mathrm{Br}<\mathrm{I}$.

From the pDoS we can calculate the vibrational energy $\left(E_{v i b}\right)$ and $S_{v i b}$. [29] By including a configurational entropy term, $S_{\text {conf }}=R \ln \Omega$, with $\Omega$ as the number of microstates, [30] which corresponds to the number of different configurations of the HX moiety in the cubic structure, and the ideal gas constant $R$, we can obtain the Gibbs free energy $G$ of both phases and predict phase transition temperatures:

$$
G=E_{N}+E_{v i b}-T\left(S_{v i b}+S_{c o n f}\right)
$$

The term $E_{N}$ is the potential energy of the crystal when atoms are at equillibrium positions. $S_{v i b}$ is calculated
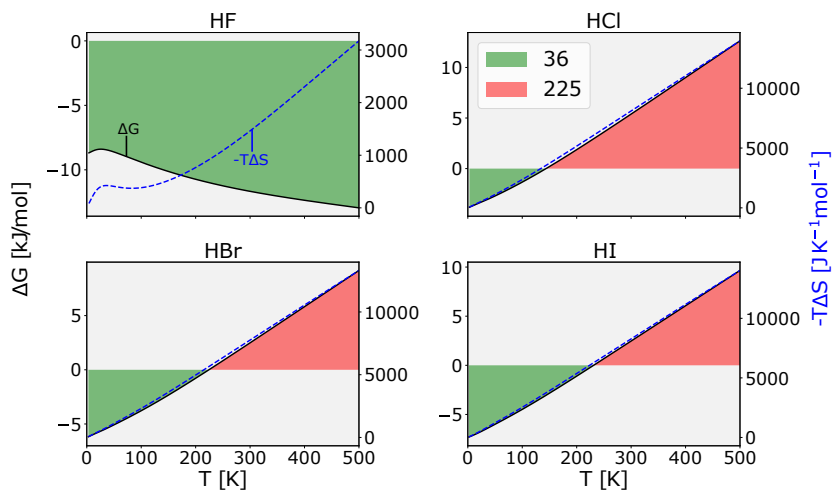

FIG. 3. Calculated difference in Gibbs free energy and difference in $-T \cdot S$ for a number of configurations of $\Omega=12$ for the high-temperature phase 225 with $\Delta G=G_{36}-G_{225}$ shown as black solid line and $\Delta S=S_{36}-S_{225}$ shown as blue dashed line. The stability region of the low-temperature phase is highlighted in green and the stability region of the high-temperature phase in red. For transition temperatures for different values of $\Omega$, see Figure S1.

from the pDoS of all phonon modes according to

$$
\begin{gathered}
S_{v i b}(T)=3 k_{B} \int_{0}^{\infty} g(\varepsilon)\{[n(\varepsilon)+1] \ln [n(\varepsilon)+1] \\
-n(\varepsilon) \ln [n(\varepsilon)]\} \mathrm{d} \varepsilon
\end{gathered}
$$

where $k_{B}$ is the Boltzmann constant, $g(\varepsilon)$ is the normalized $\mathrm{pDoS}$ with energy $\varepsilon, n(\varepsilon)=\left[\exp \left(\varepsilon / k_{B} T\right)-1\right]^{-1}$, which is the Bose-Einstein population of an energy $\varepsilon$ at temperature $T$, and $\varepsilon=\hbar \omega$ being the energy at mode frequency $\omega$. Figure 3 shows the difference in $G$, the difference in $-T \cdot S$ and the resulting stability regions of the different phases. In agreement with the experiments, no stable cubic phase is observed for HF. In the other halides, there is a phase transition from orthorhombic to cubic. $\mathrm{HCl}$ has the lowest transition temperature, followed by $\mathrm{HBr}$ and $\mathrm{HI}$, which is also in agreement with the experimental observations. In all the halide systems $-T \Delta S$ (with $\Delta S=S_{36}-S_{225}$ ) is positive and hence $\Delta S<0$, meaning that greater entropy in the 225 phase drives the phase transition $\left(S_{36}<S_{225}\right) . \Delta S$ has two contributions $\Delta S_{\text {conf }}$ and $\Delta S_{v i b} ; \Delta S_{\text {conf }}$ is always negative, and is the same for all of the systems. There is a noticeable difference in the $\Delta G$ and $\Delta S$ of $\mathrm{HF}$, where there is no predicted phase transition and the entropy difference between phases is significantly smaller than in the other hydrogen halides. This means that the $\Delta S_{v i b}$ term in HF must differ significantly from the other halides, as all other factors are constant. We now examine the vibrational modes to gain deeper insight to the origins of this difference.

The 9 lowest energy vibrational modes are classified by motion type in Figure 4 (also Table S1). Figure 5 clearly demonstrates that these modes account for almost all of 


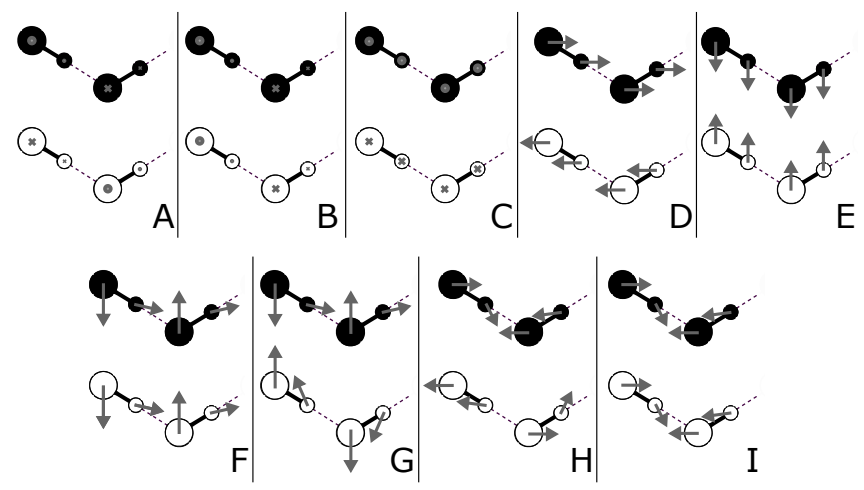

FIG. 4. Outline of the assigned modes. Black shows the atoms in the front-plane and white the atoms in the back-plane. Big atoms are halides, small are hydrogen. The dashed line indicates hydrogen bonds. Motions to the back are indicated with a cross, motions to the front with a circle. Size indicates approximate amplitude.

the vibrational entropy in the materials due to the inverse exponential dependence of entropy on the frequency. The pDoS and $S_{v i b}$ at different temperatures are given in Figure 5 with insets of the assigned modes. All other highfrequency modes are solely hydrogen motions and were not further assigned due to their negligable contribution to the vibrational entropy.

The modes in HF are more significantly more spread out than in the other hydrogen halides, especially at higher energies. For the orthorhombic phase of $\mathrm{HCl}$ two peaks in the pDoS can be observed (Figure 5) below $5 \mathrm{THz}$ which merge together when going to $\mathrm{HBr}$ and HI. This can be explained by an increase of the reduced mass of the harmonic springs, pulling the energies (frequencies) towards lower values. This trend is also seen for the modes $F, G, H$ and $I$. In $\mathrm{HCl}$ and $\mathrm{HBr}$, these modes can be clearly distinguished as separate peak in the pDoS, whilst in HI, they are energetically very close to the other modes. A similar but less pronounced trend can be seen in the high-temperature phase, see Figure S3.

$\mathrm{HF}$ has a significantly different $\mathrm{pDoS}$ with modes $F$, $G, H$ and $I$ at notably higher energies compared to the heavier HXs. These modes involve stretching or compression of intermolecular hydrogen bonds, see Figure 4. As pointed out earlier, the hydrogen bonds are very strong in $\mathrm{HF}$ compared to the other halides, or in other words, the bonds have greater force constants. In combination with a lighter reduced mass this results in the significantly higher frequency of these modes. This qualitative difference in the vibratrional frequencies of the hydrogen-halide bond is a first clue to the origin of the different phase-transition behaviour in HF; however, as the phase-transition depends on the difference $\Delta G$ between the phases, we need to look into the difference in vibrational entropy $\Delta S_{v i b}$ and hence $\Delta \mathrm{pDoS}$.
$\Delta \mathrm{pDoS}, \Delta S_{v i b}$ and the respective integrated quantity $\Delta \bar{S}_{v i b}$ with respect to the frequency are shown in Figure 6. It can be seen that in the HX systems other than HF the differences in the low frequency contributions (below $8 \mathrm{THz}$ ) of the vibrational entropy more-or-less cancel out. Comparing $\Delta \bar{S}_{v i b}$ with the assumed configurational entropy of the high-temperature phase $\left(S_{\text {conf }}=R \ln (12) \approx 20.7 \mathrm{JK}^{-1} \mathrm{~mol}^{-1}\right)$, shows that in $\mathrm{HCl}, \mathrm{HBr}$ and $\mathrm{HI}$ the influence of the vibrational entropy is indeed very small. This means, that the main phase-transition behaviour for heavier halides arises from $S_{\text {conf }}$, which supports the idea of the disorder representation of the 225 phase.

The vibrational entropy difference in $\mathrm{HF}$ has to be large to counteract the configurational entropy in order to give rise for the difference $-T \Delta S$ behaviour in Figure 3. In Figure 6 it can be seen that $\bar{S}_{v i b}$ is positive (at $300 \mathrm{~K}$ ), meaning that the low-temperature phase has a greater vibrational entropy than the high-temperature phase. Because modes F, G, H and I are of such high energy in HF that they do not contribute strongly to $S_{v i b}$. In the other halide systems, these modes are due to the weaker HX bond of lower energy and contribute to a balanced difference in vibrational entropy between phases.

These results can be used as a basis for understanding how halide exchange can be used as a handle to tune the bonding enthalpy and entropy in hydrogen-halide bonded materials. The HF bond is quantitatively and qualitatively extremely different to the other hydrogen halides. In $\mathrm{HF}$ the hydrogen bond is very strong and stiff and hence its high-frequency modes do not contribute heavily to the vibrational entropy. The strength of hydrogen bonds in HF also means it affects the balance in vibrational entropy between orthorhombic and cubic structures in $\mathrm{HF}$, revealing that the vibrational entropy is greater in the orthorhombic phase of HF than in the cubic phase, and helping to explain the lack of a solid-solid phase transition with increase temperature in this material. The heavier halides $(\mathrm{Cl}, \mathrm{Br}, \mathrm{I})$ all have hydrogen bond modes that are low enough in frequency to have a significant contribution to vibrational entropy at room temperature and below. The vibrational entropy contribution in the heavier halides is relatively similar across the series and it has also been shown that the hydrogen bond strength does not vary significantly with the heavier halides. These have a greater influence from dispersive forces and less from their weaker hydrogen bonds.

In the context of materials with hydrogen-halide bonds like HOIPs, where the balance of hydrogen bond strength to vibrational entropy has been shown to have profound effects on phase transitions, [4] these findings are important for targeted materials design. These results indicate that substitution of heavier halides can be executed with little effect on the energies of phase transitions in HOIPs to achieve other property changes, for example band gap tuning.[31, 32] On the contrary 

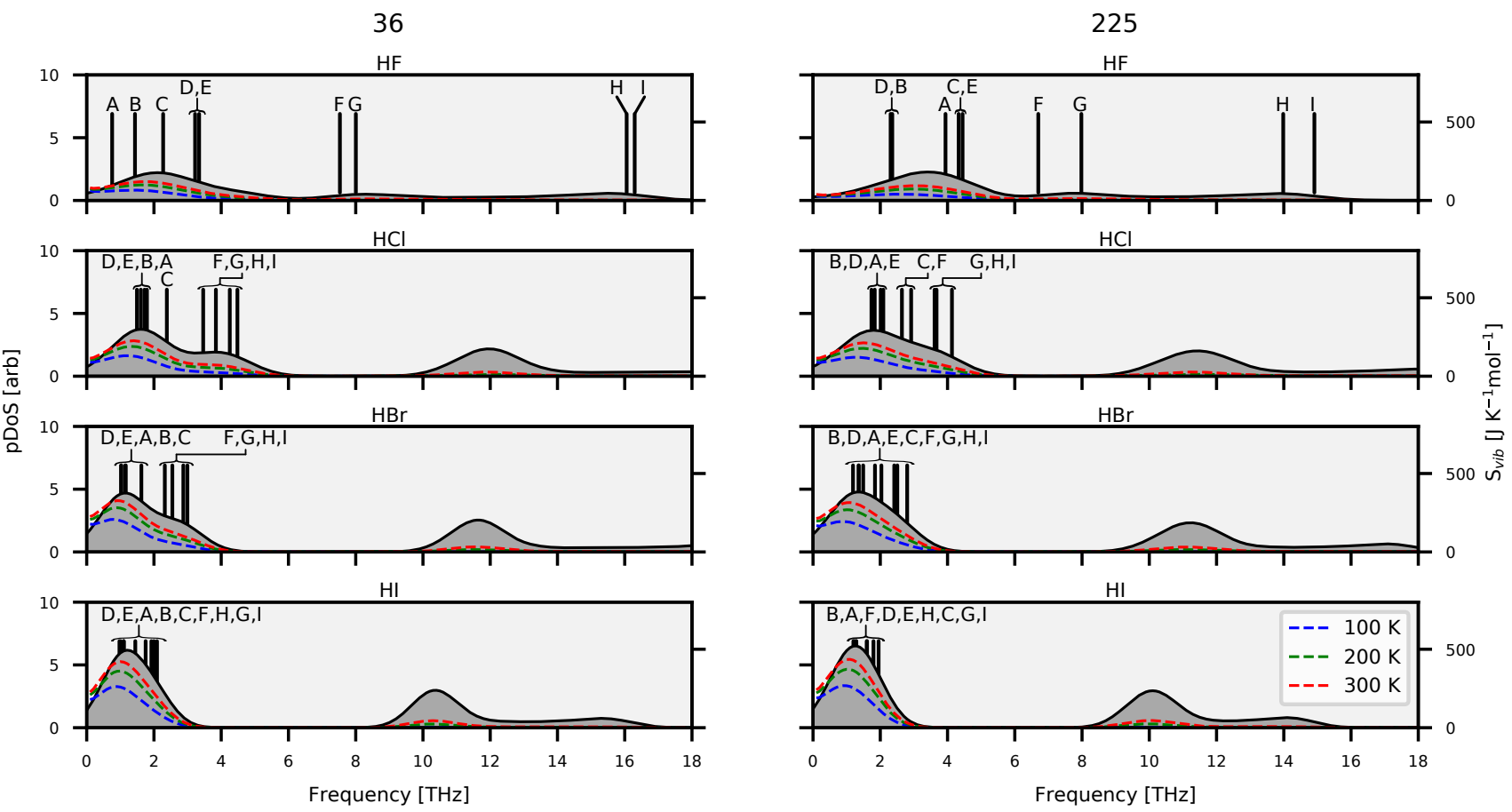

FIG. 5. pDoS (black) and $S_{v i b}$ for $100 \mathrm{~K}, 200 \mathrm{~K}$ and $300 \mathrm{~K}$ (dashed lines) of the two phases. The locations of the different modes depicted in Figure 4 are indicated on the plots.

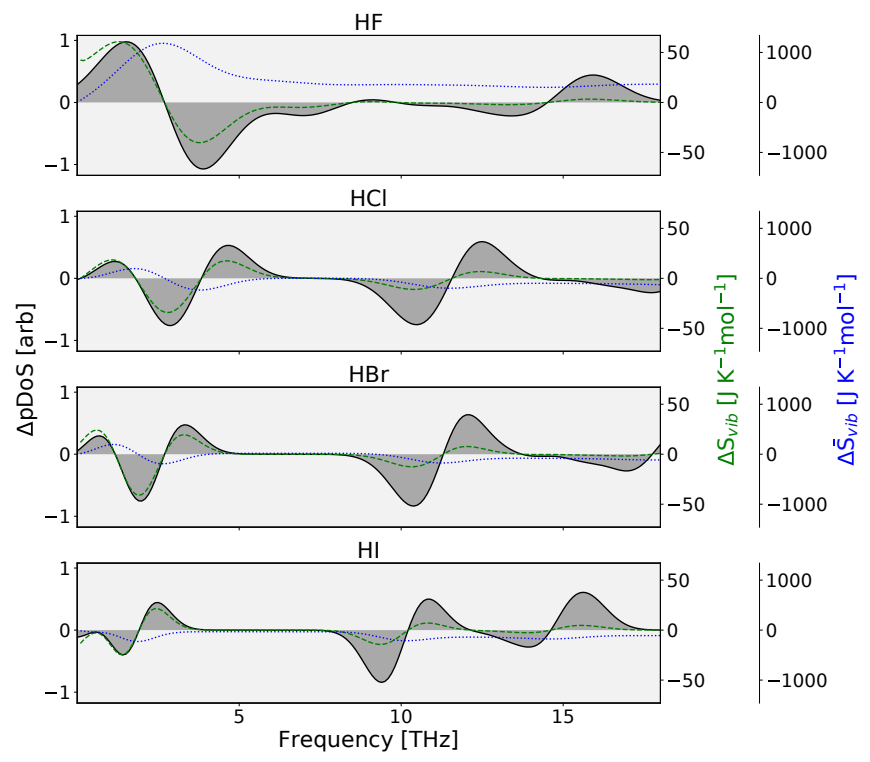

FIG. 6. Difference of the pDoS depicted as solid black line, difference of $S_{v i b}$ (green) $\left(\Delta S_{v i b}=S_{v i b_{36}}-S_{v i b_{225}}\right)$ as dashed green line and integrated difference of vibrational entropy $\Delta \bar{S}_{v i b}$ as dotted blue line, calculated at $300 \mathrm{~K}$.

F substitution can be used to tune phase stabilities significantly.

In conclusion we have presented a series of first principles calculations to probe the entropy/enthalpy relation- ship in hydrogen-halide bonds. We calculated vibrational entropy and analysed this in terms of the normal modes of the systems. This analysis of simple binary systems allows us to draw inferences which can be hidden in more complex multi-component systems. The principles uncovered, however have implications for materials design in these more complex systems, and tuning of phase transition temperatures and other physical properties (e.g. optical absorption or emission) can be decoupled by judicious choice of halide anions.

\section{METHODS}

\section{Generating structures}

As discussed earlier, there are or might be several phases associated with different hydrogen halides. The low-temperatures structure (space group number 36) is well defined and X-ray diffraction (XRD) data is often available. However, the other phases might have disorder and hence have no simple way to represent them. The cubic high-temperature phase with the space group number 225 has for example in the XRD-data a rocksalt structure. The crystallographic structure is most probably a dynamic average of disorderd $\mathrm{H}$ positions. To achieve a static representations the hydrogen atoms were displaced so that they recreate the chain-structure in the low-temperature phase, but with the halide atoms are still on fcc positions, see Figure 7. Table S2 summarises 


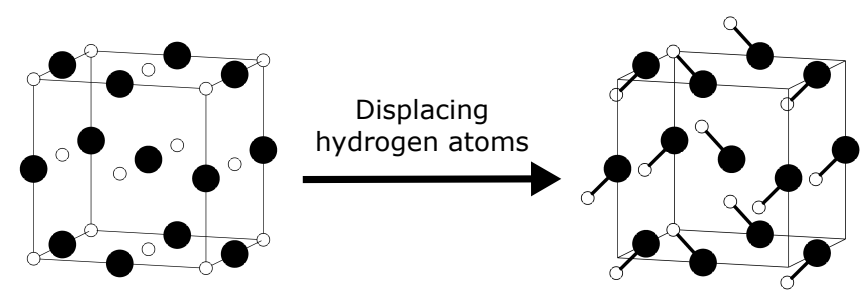

FIG. 7. Displacement of the hydrogen atoms from a rocksaltstructure to a cubic pseudo 36 structure (color code: black halide, white - hydrogen).

the sources and the methods with which each of the structures was generated.

\section{DFT calculations}

All DFT calculations were performed with the Vienna Ab Initio Simulation Package (VASP) [33] with the Generalized Gradient Approximation (GGA) level with a PBE functional.[34] An energy-cutoff of $650 \mathrm{eV}$, k-spacing of 0.15 and an energy-difference as stopping condition for the SCF cycle of $1.00 \mathrm{e}-6$ was chosen. Additionally, the force-difference stopping criterion for the relaxation was set to $1.00 \mathrm{e}-2$ and a DFT-D3 method with Becke-Jonson damping was used as vdW correction.[35] First, ions were relaxed, then the ions and the cell shape and finally the ions, the cell shape and the cell volume. In order to sustain the cubic symmetry, the procedure for the cubic 225 phase was changed. Here, ions were relaxed first and then only the cell volume.

For the phonon calculations, $2 \times 2 \times 2$ supercells were used. Displacements were generated with PHONOPY(version 1.40) with a displacement amplitude of $0.01 \AA$.[36] The single displaced structures were calculated with VASPwith one ionic relaxation step.

The calculation of the $\mathrm{pDoS}$, vibrational entropy and free energy were performed by using a $8 \times 8 \times 8$ mesh in PHONOPY. We would like to note that with GGA level calculations in the closely related systems $\mathrm{Br}_{2}$ and $\mathrm{I}_{2}$ erroneous dynamic instable ground state structures were found due to high delocalization.[37] However, as HX systems are rather localized, dispersion corrected GGA calculations are sufficient, also indicated by the absence of imaginary modes.

\section{ACKNOWLEDGEMENTS}

D.B. acknowledges his scholarship from the Studienstiftung des Deutschen Volkes.

\section{REFERENCES}

[1] A. K. Cheetham, G. Kieslich, and H. H.-M. Yeung, Acc. Chem. Res. 51, 659 (2018).

[2] A. P. Katsoulidis, D. Antypov, G. F. S. Whitehead, E. J. Carrington, D. J. Adams, N. G. Berry, G. R. Darling, M. S. Dyer, and M. J. Rosseinsky, Nature 565, 213 (2019).

[3] Y. Shchur, Phase Transit. 86, 857 (2013).

[4] G. Kieslich, J. M. Skelton, J. Armstrong, Y. Wu, F. Wei, K. L. Svane, A. Walsh, and K. T. Butler Chem. Mater. 30, 8782 (2018),.

[5] A. M. Walker, B. Civalleri, B. Slater, C. MellotDraznieks, F. Corà, C. M. Zicovich-Wilson, G. RománPérez, J. M. Soler, and J. D. Gale, Angew. Chem. Int. Ed. 49, 7501 (2010).

[6] W. Li, Z. Wang, F. Deschler, S. Gao, R. H. Friend, and A. K. Cheetham, Nat. Rev. Mater. 2, 16099 (2017).

[7] M. Ruggiero, J. Infrared Millim. Terahertz Waves , 1-38 (2020).

[8] M. R. Ryder, J. Maul, B. Civalleri, and A. Erba, Adv. Theory Sim. 2, 1900093 (2019).

[9] E. Cockayne, J. Phys. Chem. C 121, 4312 (2017).

[10] S. Ling and B. Slater, Chem. Sci. 7, 4706 (2016).

[11] J. Wieme, K. Lejaeghere, G. Kresse, and V. V. Speybroeck, Nat. Commun. 9 (2018), 4899.

[12] K. T. Butler, P. Vervoorts, M. G. Ehrenreich, J. Armstrong, J. M. Skelton, and G. Kieslich, Chemistry of Materials 31, 8366 (2019).
[13] T. D. Bennett, A. K. Cheetham, A. H. Fuchs, and F.-X. Coudert, Nat. Chem. 9, 11 (2017).

[14] T. Steiner, Angew. Chem. Int. Ed. 41, 48 (2002).

[15] M. Atoji and W. N. Lipscomb, Acta Cryst. 7, 173 (1954).

[16] M. W. Johnson, E. Sándor, and E. Arzi, Acta Cryst. B 31, 1998 (1975).

[17] E. Sándor and R. F. C. Farrow, Nature 213, 171 (1967).

[18] E. Sándor and M. Johnson, Nature 217, 541 (1968).

[19] G. Natta, Gazzetta Chimica Italiana 63, 425 (1933).

[20] E. Sándor and R. F. C. Farrow, Nature 215, 1265 (1967).

[21] N. Niimura, K. Shimaoka, H. Motegi, and S. Hoshino, J. Phys. Soc. Jpn. 32, 1019 (1972).

[22] H. Chihara and A. Inaba, J. Chem. Thermodyn. 8, 935 (1976).

[23] J. Cockcroft, A. Simon, and K. R. A. Ziebeck, Z. Kristallogr. Cryst. Mater. 184, 229 (1988).

[24] A. Simon, Z. Naturf. 25b, 1489 (1970).

[25] A. Ikram, B. Torrie, and B. Powell, Mol. Phys. 79, 1037 (1993).

[26] W. Zeng, Y. Mao, and A. Anderson, J. Raman Spectrosc. 30, 995 (1999).

[27] K. L. Svane, A. C. Forse, C. P. Grey, G. Kieslich, A. K. Cheetham, A. Walsh, and K. T. Butler, J. Phys. Chem. Lett. 8, 6154 (2017).

[28] W. Haynes, CRC Handbook of Chemistry and Physics, CRC Handbook of Chemistry and Physics (CRC Press, 2014).

[29] B. Fultz, Prog. Mater. Sci. 55, 247 (2010).

[30] P. Atkins and J. de Paula, Atkins' Physical Chemistry (Oxford University Press, 2009). 
[31] G. E. Eperon, S. D. Stranks, C. Menelaou, M. B. Johnston, L. M. Herz, and H. J. Snaith, Energy Environ. Sci. 7, 982 (2014).

[32] J. H. Noh, S. H. Im, J. H. Heo, T. N. Mandal, and S. I. Seok, Nano Lett. 13, 1764 (2013), pMID: 23517331.

[33] G. Kresse and J. Furthmüller, Phys. Rev. B 54, 11169 (1996).
[34] J. P. Perdew, K. Burke, and M. Ernzerhof, Phys. Rev. Lett. 77, 3865 (1996).

[35] S. Grimme, S. Ehrlich, and L. Goerigk, J. Comput. Chem. 32, 1456 (2011).

[36] A. Togo and I. Tanaka, Scr. Mater. 108, 1 (2015).

[37] J. George, C. Reimann, V. L. Deringer, T. Bredow, and R. Dronskowski, ChemPhysChem 16, 728 (2015). 